\title{
INOVAÇÃO EM DESIGN DE MODA: UMA REVISÃO BIBLIOMÉTRICA DA LITERATURA
}

Irina Lopes Guedes

Mestranda em Design, Universidade Federal de Santa Catarina.

Irina.lopes@gmail.com

Giselle Schmidt Alves Díaz Merino, Dra

Dra., Universidade Federal de Santa Catarina.

gisellemerino@gmail.com

Eugenio Andrés Díaz Merino, Dr.

Universidade Federal de Santa Catarina.

eugenio.merino@ufsc.br

Luiz Salomão Ribas Gomez, PhD

Universidade Federal de Santa Catarina.

salodesigner@gmail.com

Resumo: Os processos de inovação vêm sendo abordados por diversas áreas. Dentre elas o Design de moda se apresenta com um grande potencial, entretanto o número de pesquisas científicas que abordam a inovação neste setor é considerado baixo. Pretende-se levantar e identificar as pesquisas que focam na inovação no processo de desenvolvimento de produtos da área da moda, por meio de revisão bibliométrica. Nesse ínterim, cabe conceituar Inovação, buscando apresentar a relevância do tema no setor de moda, fazendo a associação do mesmo às pesquisas de tendências. Sendo assim, uma pesquisa exploratória permitiu a definição dos termos "Inovação", "Desenvolvimento de produto", "Pesquisa e Desenvolvimento", "Design", "Moda" e "Tendências", bem como a construção dos comandos de busca utilizados. A revisão foi realizada no dia 06 de junho de 2016, na base ProQuest (New Technology and Engineering), na qual se obteve o resultado de 165 trabalhos, e na base Scopus, onde foram encontrados 7275 trabalhos, sobre Inovação e Design de Moda em ambas as bases. Estes números mostraram não estar de acordo com as premissas da pesquisa, sobretudo na base Scopus, onde os resultados misturavam-se com conteúdos diversos, de acordo com revisão aleatória feita a partir dos títulos. Cabe, dessa forma, dar seguimento à pesquisa selecionando e analisando os trabalhos de fato pertinentes ao tema. Este trabalho, portanto, ateve-se a analisar os resultados da base ProQuest, apresentando os resultados em número de publicações por década e sua distribuição entre os periódicos. 
Palavras-chave: Revisão bibliométrica. Inovação. Design de Moda.

\begin{abstract}
Innovation processes have been approached by several areas. Among them the Fashion Design area shows great potential, but the number of scientific research addressing innovation in this sector is considered low. This work intends to raise and identify researches that focus on innovation in the process of fashion product development through bibliometric review. In the meantime, it conceptualizes Innovation, seeking to show the relevance of the theme in the fashion industry and making the association of it with trends researches. An exploratory study allowed the definition of the terms "Innovation", "Product Development", "Research and Development", "Design", "Fashion" and "Trends", as well as the construction of the search commands used. The review was held on June 6, 2016, in ProQuest base (New Technology and Engineering), in which it obtained the result of 165 works, and Scopus, where 7275 works were found. These figures showed not conformation to the definitions of the research. This happened particularly in Scopus where the results mingled with various contents, according to random review based on work titles. It is, therefore, apropriate to proceed with the search by selecting and analyzing the papers indeed related to the theme. This work focused on analyzing the results of ProQuest base, presenting the results in the number of publications per decade and its distribution among the journals.
\end{abstract}

Keywords: Bibliometric Review. Innovation. Fashion Design.

\title{
1. INTRODUÇÃO
}

O setor de moda é visto como um dos mais importantes setores da indústria brasileira. De acordo com site Design Brasil (2013) ${ }^{1}$ "o setor têxtil é responsável por $17,5 \%$ do PIB da indústria de transformação nacional e por cerca de 3,5\% de todo o PIB do Brasil." Além disso, o setor movimenta em torno de US\$ 46 bilhões ao ano e emprega 1,65 milhão de trabalhadores, com uma cadeia produtiva de aproximadamente 30 mil empresas. A Associação Brasileira da Indústria Têxtil e de Confecção - ABIT (2013) demonstra que apenas no ano de 2012 o BNDES (Banco Nacional do Desenvolvimento) investiu $1.478,5$ milhões de reais somente na indústria de confecção.

Essa movimentação não envolve unicamente as empresas que desenvolvem o produto final do setor de moda, mas está relacionada também aos setores de marketing, branding e distribuição, além da produção dos suprimentos para o setor, tais como tecidos, aviamentos e beneficiamentos.

1 “O portal Design Brasil é uma iniciativa do Ministério do Desenvolvimento Indústria e Comércio Exterior (MDIC), através do Programa Brasileiro de Design (PBD) em conjunto com o SENAI e o SEBRAE. O objetivo é promover a integração, a convergência e cooperação entre as diversas ações na área do design em todo o país." (DESIGNBRASIL, 2013) 
A importância do setor, a demanda constante por novidades por parte dos consumidores de moda e a necessidade de permanência das empresas no mercado apontam a relação direta entre Inovação e o mercado de moda.

Apesar dos processos de inovação vir sendo amplamente estudados - em especial nas áreas das Engenharias, mas também na área do Design, uma breve pesquisa exploratória traz à luz o baixo número de pesquisas que abordem a Inovação em Design de Moda.

Assim, o objetivo deste trabalho é levantar e identificar as pesquisas que focam na inovação no processo de desenvolvimento de produtos da área da moda, fazendo uso da técnica revisão bibliométrica, que "estuda as características externas de documentos de uma área da literatura especializada" (BUFREM; PRATES, 2005, p. 13).

A presente pesquisa tem natureza teórica, de abordagem mista (quantitativa e qualitativa), quanto à apresentação dos dados, e se caracteriza como revisão bibliográfica em relação aos procedimentos técnicos. Por buscar conhecer o melhor o problema por meio de estudos já realizados, classifica-se como exploratória e também descritiva, pois busca estabelecer relações entre os dados (GIL, 2002).

Dessa forma, os primeiros passos para o desenvolvimento da revisão foram a realização de uma pesquisa exploratória sobre o tema no Portal CAPES e Google Scholar, e a construção de um protocolo de pesquisa. Neste protocolo, definiu-se o objetivo previamente citado, e a principal questão a ser respondida pela pesquisa, sendo Q: Qual o volume de trabalhos científicos que abordam Inovação e Design de Moda, onde estão distribuídos e em que tipo de trabalho se enquadram. Também foram especificadas neste documento as estratégias de busca a serem utilizadas, definindo-se os termos-chave como "Inovação", "Desenvolvimento de produto", "Pesquisa e Desenvolvimento", "Design", "Moda" e "Tendências", bem como critérios para inclusão e exclusão de trabalhos, entre outros itens delimitadores.

A revisão foi realizada nas bases de dados ProQuest (New Technology and Engineering), e Scopus, apontadas na pesquisa exploratória como algumas das mais relevantes bases de dados sobre o tema.

\section{CONSIDERAÇÕES INICIAIS}

\subsection{A compreensão sobre Inovação}

Parece haver consenso entre diversos autores sobre a situação do termo "Inovação" nas organizações, no sentido de que se discute muito sobre o tema, mas frequentemente não se sabe de fato o que "Inovação" significa.

Kelley e Littman $(2007$, p.3) são pragmáticos ao dizer que "a inovação é a seiva de todas as organizações". Tal a afirmação não é à toa, pois o consumo atual está pautado na ideia de que as novidades são sempre uma evolução natural dos produtos, e são, portanto, melhores que os seus representantes anteriores. Além disso, o sistema capitalista se sustenta através do consumo, tornando este pensamento essencial para a permanência das empresas do mercado. Assim, entende-se que o incentivo ao consumo determina a sobrevivência das empresas no mercado; o consumo, por sua vez, está muitas vezes condicionado à ideia do novo sendo algo melhor. Dessa forma, fica evidente a importância da inovação no desenvolvimento de novos produtos.

Lynn e Reilly (2003, p.15) corroboram o exposto ao afirmar que "grandes empresas alcançam sucesso e longevidade porque geram novos produtos 
continuamente. Empresas fracassam porque se esquecem dessa verdade simples". Porém, os autores enfatizam a necessidade de geração de valor real aos consumidores e propõem que as equipes de desenvolvimento de novos produtos que obtiveram sucesso atendiam a cinco práticas essenciais. Estas práticas envolvem a cooperação total dos mais altos níveis de gerência; estabelecimento de fundamentos de projeto desde o início, trazendo visão estável e nítida; improvisação; troca de informações feita além das reuniões formais, de forma ampla e intensa; e por fim a colaboração sob pressão (LYNN; REILLY, 2003).

Estas práticas não parecem ser suficientes, na visão de Kotler (2011), para solucionar as barreiras à Inovação. A atribuição imprecisa de responsabilidade pela inovação é um dos problemas apontados pelo autor, citando-a como o "sem-teto" da empresa, uma vez que os setores de Pesquisa e Desenvolvimento (P\&D) passam a responsabilidade, sobre as inovações não tecnológicas, para os setores de marketing. Estes por sua vez estão centrados no marketing da continuidade e não no marketing de inovação. Ou, ainda, quando as responsabilidades são divididas, não há um gerenciamento estratégico para o alcance dos resultados (KOTLER, 2011).

Apesar de muitos autores dissertarem também sobre as diferenças entre inovação disruptiva, inovação incremental, inovação gradativa e inovação radical, este trabalho se aterá aos conceitos de inovação apresentados por Baregheh, Rowley e Sambrook (2009) e pelo Manual de Oslo (FINEP, 1997). Tais conceitos foram selecionados por apresentarem definições amplas que abarcam muitos dos pressupostos apresentados pelos demais autores:

\begin{abstract}
Innovation is the multi-stage process whereby organizations transform ideas into new/improved products, service or processes, in order to advance, compete and differentiate themselves successfully in their marketplace. ${ }^{2}$ (BAREGHEH, ROWLEY e SAMBROOK, 2009).

Uma inovação é a implementação de um produto (bem ou serviço) novo ou significativamente melhorado, ou um processo, ou um novo método de marketing, ou um novo método organizacional nas práticas de negócios, na organização do local de trabalho ou nas relações externas. (FINEP, 1997).
\end{abstract}

Uma vez apresentadas as definições sobre Inovação faz-se necessário observar o setor de Moda, bem como os aspectos do Design no setor, a fim de compreender o papel da Inovação no mesmo.

\title{
2.2. Inovação em Design de Moda
}

Graças à notoriedade expressa nos números já apresentados do setor têxtil e de confecção no Brasil, cresceu junto com o setor a necessidade de atender a avidez dos consumidores por novidades, demanda inerente ao sistema de moda.

Essa necessidade parece estar presente em todos os níveis da cadeia de produção, desde empresas do comércio de varejo até empresas de fiações e tecelagens. Assim, toda a cadeia em torno de produtos de moda busca de alguma maneira atualizar-se e trazer ao mercado alguma forma de inovação.

2 Inovação é um processo de várias etapas através do qual organizações transformam idéias em novos/melhores produtos, serviços ou processos, com o intuito de avançar, competir e diferenciar-se com sucesso em seu mercado. (BAREGHEH, ROWLEY e SAMBROOK, 2009) 
Cachon e Swinney (2011) afirmam que muitas empresas do setor têm adotado o sistema de fast fashion, que combina um curto tempo de produção e distribuição ao design de produtos com alto teor de informação de moda, chamados trendy products.

Nesse ínterim, entram em cena as pesquisas de tendências, que buscam diminuir o gap entre os desejos dos consumidores e as ofertas de produtos e serviços por parte das empresas. Caldas (2004) afirma que:

[...] é imperativo que o homem especule sobre o que está por vir, pois fazer previsões é uma forma de controlar a vida e de confrontar a experiência da morte que trazemos no inconsciente. Por outro lado, quanto mais complexa se torna a sociedade, maior a necessidade de planejar e prever e, ao mesmo tempo, mais difícil. Daí a utilidade de um conceito que permite construir uma ponte sobre esse lapso de tempo, ainda mais se essa ponte predefine uma direção, como ocorre com as idéias de evolução e progresso (CALDAS, 2004. P.35,36).

O autor traz também uma definição breve e direta sobre Tendência, colocandoa como "manifestação, na esfera do comportamento, do consumo ou do 'espírito do tempo', de uma sensibilidade anunciada por sinais. Para uma cadeia produtiva, adquire o sentido de redutor de risco" (CALDAS, 2004. P.217). Assim, é possível compreender que as pesquisas de tendências buscam justamente proporcionar às empresas o conhecimento necessário sobre os hábitos do mercado e dos consumidores, a fim de embasar o processo de inovação de produtos de moda e adiantar-se às necessidades do mercado.

De acordo com Sousa, Gomez e Campos (2013, p.37):

De maneira sistemática ou não, nos estudos e nos negócios da moda, há o
interesse pela prospecção de tendências, as quais possam indicar o que está
acontecendo e antecipar o que irá influenciar o modo de vestir e o
comportamento das pessoas. Em torno disso há diferentes interesses que,
de maneira direta ou indireta, implicam em retorno ou lucro financeiro.

Estas colocações demonstram a importância da pesquisa de tendências para o setor, bem como a estreita relação entre Inovação e a prospecção de tendências. Desta forma, este trabalho busca levantar e identificar as pesquisas que foquem na inovação no processo de desenvolvimento de produtos da área da moda, por meio de revisão bibliométrica, buscando identificar as associações entre os termos Inovação e Tendências em Design de Moda.

\section{REVISÃO BIBLIOMÉTRICA DA LITERATURA}

Revisões bibliométricas exigem inicialmente uma pesquisa exploratória sobre o tema, a fim de verificar termos comumente utilizados, e a construção de um protocolo de pesquisa. Neste protocolo, apresenta-se o objetivo da pesquisa e as questões às quais a mesma deve responder. Assim, para alcançar o objetivo, a pesquisa deverá responder a seguinte questão de investigação: Q) Qual o volume de trabalhos científicos abordando Inovação e Design de Moda, onde estão distribuídos e em que tipo de trabalho se enquadram.

A pesquisa exploratória foi realizada inicialmente no portal da CAPES e no Google Scholar, buscando os termos "Inovação", "Design" e "Moda". No dia 20 de 
maio de 2016, os resultados encontrados com a inserção somente da palavra "Inovação" incluíam 6.931 resultados. Estreitou-se a busca limitando-a às áreas de Business and Economics, das Sociais Aplicadas: Antropologia, e das Engenharias: Material Science. Também restringiu-se nesse momento o período de publicação, limitando-o,como ano mais antigo, o ano de 2006. Estas definições diminuíram os resultados para 4.808 trabalhos. Um novo refinamento foi feito a partir de tópicos, que excluíam os termos Brazil, Latin America, Entrepeneurship, Competitividade, Competitiveness e Sustainable development, e a partir de coleções, excluindo Medline/Pubmed, Civil Engineering Abstracts e Mechanical and Transportation Engineering Abstracts, todos por apresentarem resultados não compatíveis com o tema da busca, restando então 4.316 trabalhos. O passo seguinte foi iniciar uma nova busca utilizando os termos "Inovação" e "Design". Esta busca apresentou resultado menos significativo em número, com apenas 321 artigos. Nessa busca também foram excluídas coleções: Medline/Pubmed, Solid States and Superconductivity Abstracts e ASFA: Acquatic Science and Fisheries Abstracts. Por fim, incluiu-se o termo "Moda" à pesquisa, associando-o à "Inovação" e "Design", resultando em apenas 11 trabalhos. A partir de alguns dos artigos encontrados nos resultados descritos acima, identificaramse os termos mais comumente utilizados nas pesquisas da área em português. Estes termos foram traduzidos para o idioma inglês, buscando também suas variações. Em seguida, os termos foram ajustados para a pesquisa nas bases, utilizando truncamento e booleanos, conforme o quadro abaixo (quadro 1). Dos termos em inglês, apenas a palavra Fashion foi removida, pois possui significado diverso ao interesse desta pesquisa quando utilizado individualmente.

Quadro 1: Palavras-chave utilizadas

\begin{tabular}{|c|c|c|}
\hline PORTUGUÊS & INGLÊS & TRUNCAMENTO E BOOLEANOS \\
\hline Inovação & $\begin{array}{l}\text { Innovation, Innovation process, } \\
\text { Innovative products, } \\
\text { innovator products, Innovation } \\
\text { projects, Design driven innovation }\end{array}$ & $\begin{array}{l}\text { (Innovation OR "innovat* process*" } \\
\text { OR "innovat* product*" OR } \\
\text { "innovat* project\$” OR "design } \\
\text { driven innovation") }\end{array}$ \\
\hline $\begin{array}{l}\text { Desenvolvimento de } \\
\text { produto }\end{array}$ & $\begin{array}{l}\text { Product development, product } \\
\text { design }\end{array}$ & $\begin{array}{l}\text { (“product\$ develop* OR “product\$ } \\
\text { design") }\end{array}$ \\
\hline $\begin{array}{l}\text { Pesquisa e } \\
\text { Desenvolvimento }\end{array}$ & Research and Development, R\&D & $\begin{array}{l}\text { (“Research and development" OR } \\
\text { "R\&D") }\end{array}$ \\
\hline Design & Design, product design & (design OR “product\$ design") \\
\hline Moda & $\begin{array}{l}\text { fashion (removida por trazer } \\
\text { resultados incoerentes.), Fashion } \\
\text { design, Fashion Industry, Textile, } \\
\text { textile design }\end{array}$ & $\begin{array}{l}\text { (textile\$ OR “fashion design" OR } \\
\text { "textile\$ design" OR "fashion } \\
\text { industr*" OR "textile\$ industr*”) }\end{array}$ \\
\hline Tendências & $\begin{array}{l}\text { Trends, fashion trends, market } \\
\text { trends, diffusion, fashion diffusion, } \\
\text { fashion forecaster }\end{array}$ & $\begin{array}{l}\text { (trend* OR “fashion trend\$” OR } \\
\text { "market trend\$” OR diffus* OR } \\
\text { "fashion diffus*" OR "fashion } \\
\text { forecast*") }\end{array}$ \\
\hline
\end{tabular}

Fonte: Elaborado pelo autor, com base na pesquisa realizada.

A pesquisa exploratória também permitiu identificar as bases de dados com maior volume de publicações na área, das quais se optou pelas bases ProQuest e Scopus. Na base ProQuest, a área definida a partir do mesmo critério foi Abstracts in New Technology and Engineering. 
Ainda na construção do protocolo de pesquisa foram feitas as possíveis associações entre os truncamentos e booleanos já definidos. As associações foram feitas de forma a sempre incluir os termos relacionados à Inovação e à Moda, resultando em dez diferentes combinações apresentadas no quadro 2. Estas combinações foram os comandos de busca aplicados um a um, em cada uma das bases. Cada base possui suas peculiaridades, logo, além dos comandos de busca já determinados, acertos específicos foram realizados nas duas bases para que buscassem resultados dentro dos parâmetros determinados no protocolo. Para ambas as bases limitou-se a pesquisa à arquivos em inglês, português e espanhol, e às áreas business, engineering, sociais aplicadas e artes. Também se limitou a artigos e Conference papers.

Os resultados obtidos em cada uma das bases, por meio de cada um dos comandos de busca, foram somados e posteriormente rastreados para identificação de documentos repetidos. Assim, os números finais de documentos foram reduzidos, restando somente documentos únicos.

A base Scopus foi a que mais apresentou resultados, com um total de 16.244 documentos, sendo 7.275 documentos não repetidos. Desses, 1116 estão cadastrados como Conference Paper, havendo sido publicados em eventos diversos, e 6159 como artigos publicados em periódicos.

Já a base ProQuest (ANTE) apresentou somente 226 trabalhos, dos quais 165 não se repetiam. Estes documentos encontram-se todos cadastrados como artigos publicados em periódicos.

Seguem abaixo os resultados obtidos em cada uma das bases, bem como a soma dos resultados totais e parciais, repetidos e não repetidos (quadro 2).

Quadro 2: Combinações possíveis de palavras-chave (comandos de busca), sempre incluindo termos relacionados à Inovação e Moda.

\begin{tabular}{|c|c|c|c|}
\hline COMANDOS DE BUSCA & $\begin{array}{l}\text { PROQUEST } \\
\text { (ANTE) }\end{array}$ & SCOPUS & TOTAL \\
\hline $\begin{array}{l}\text { 1. (Innovation OR “innovat* process*" OR “innovat* product*" OR } \\
\text { "innovat* project\$” OR "design driven innovation") AND (textile\$ OR } \\
\text { "fashion design" OR "textile\$ design" OR "fashion industr*" OR } \\
\text { "textile\$ industr*") }\end{array}$ & 176 & 2157 & 2333 \\
\hline $\begin{array}{l}\text { 2. (Innovation OR “innovat* process*" OR "innovat* product*" OR } \\
\text { "innovat* project\$" OR “design driven innovation") AND ("product\$ } \\
\text { develop*" OR "product\$ design") AND (textile\$ OR "fashion design" } \\
\text { OR "textile\$ design" OR "fashion industr*" OR "textile\$ industr*") }\end{array}$ & 6 & 1362 & 1368 \\
\hline 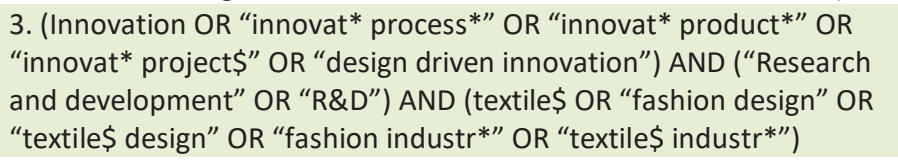 & 5 & 2037 & 2042 \\
\hline $\begin{array}{l}\text { 4. (Innovation OR "innovat* process*" OR “innovat* product*" OR } \\
\text { "innovat* project\$" OR “design driven innovation") AND (design OR } \\
\text { "product\$ design") AND (textile\$ OR “fashion design" OR "textile\$ } \\
\text { design" OR "fashion industr*" OR "textile\$ industr*") }\end{array}$ & 36 & 4075 & 4111 \\
\hline 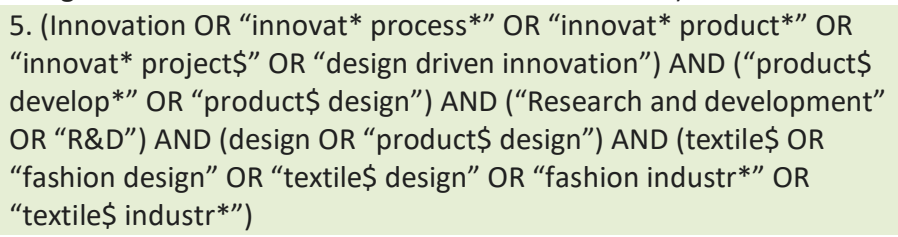 & 0 & 332 & 332 \\
\hline $\begin{array}{l}\text { 6. (Innovation OR “innovat* process*" OR “innovat* product*" OR } \\
\text { "innovat* project\$" OR “design driven innovation") AND (textile\$ OR } \\
\text { "fashion design" OR "textile\$ design" OR "fashion industr*" OR }\end{array}$ & 7 & 2704 & 2711 \\
\hline
\end{tabular}




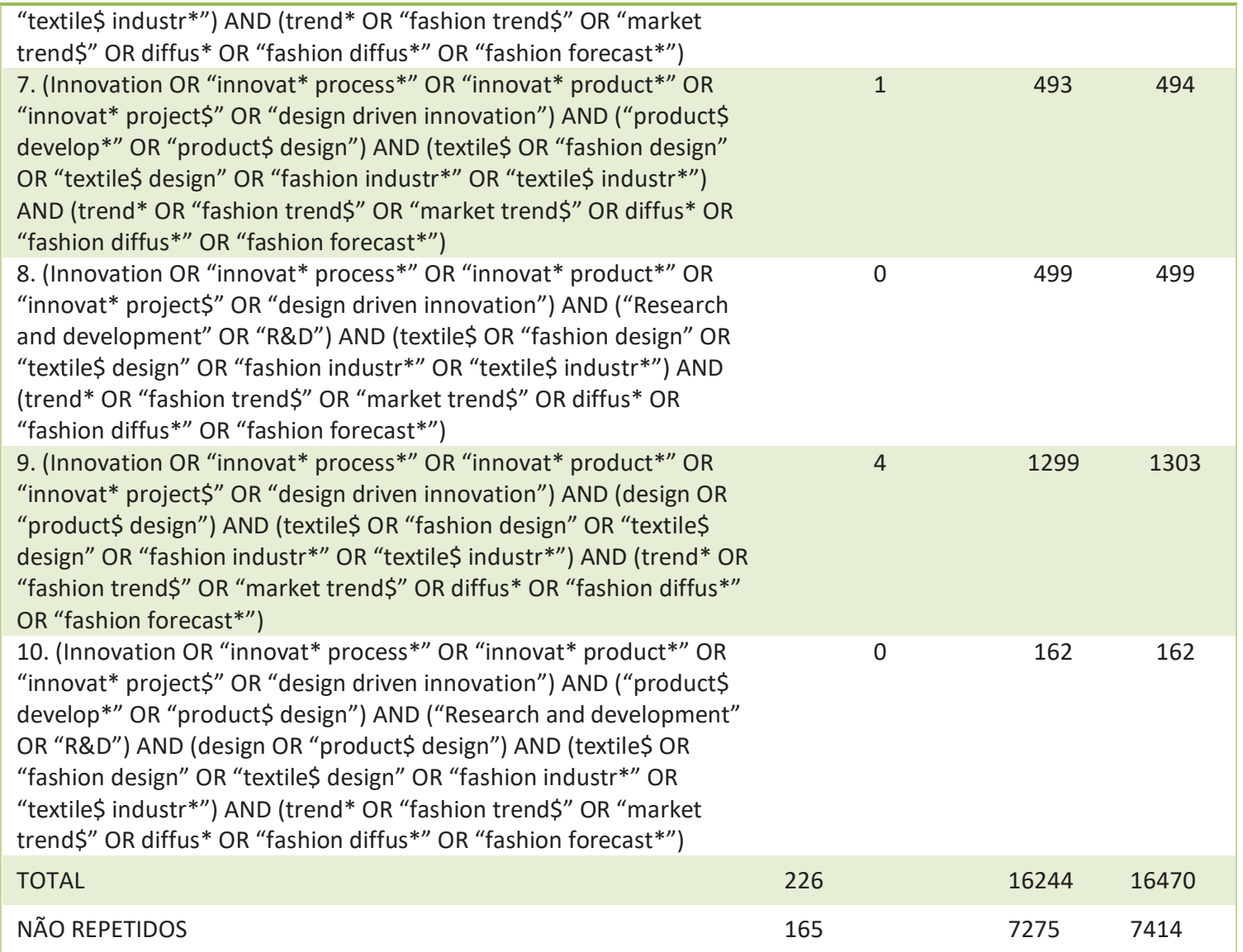

Fonte: Elaborado pelo autor, com base na pesquisa realizada.

Após a finalização das buscas nas bases de dados, ficou visível a diferença entre os resultados na base Scopus e na base ProQuest. Essa diferença em torno de sete mil trabalhos a mais na base Scopus indicou a necessidade de verificação destes trabalhos. Assim, foi feita uma revisão aleatória através dos títulos, com o intuito de confirmar a adequação dos resultados aos critérios definidos inicialmente. Nesta revisão constatou-se que parte das pesquisas apresentadas nos resultados desta base estava em desacordo com os critérios de inclusão e exclusão, mostrando a necessidade de adequação dos termos para esta base e invalidando, desta forma, estes resultados para esta pesquisa.

Dessa forma, optou-se por analisar somente os resultados da base ProQuest, pois se mostraram mais adequados. Os 165 trabalhos resultantes desta base foram analisados pelo ano de publicação e pela distribuição dentre os periódicos citados.

As duas pesquisas mais antigas datam de 1975 e foram ambas publicadas no periódico Journal of Industrial Textiles, sendo as únicas pesquisas publicadas nesta década e apresentadas pela base.

As décadas de 80 e 90 se mostraram mais férteis, apresentando 26 e 24 resultados, respectivamente. A partir dos anos 2000 as publicações aumentam e passam a corresponder a 51 trabalhos e, posteriormente, a partir de 2010, somam 62 artigos. (Gráfico 1) 


\section{Análise por ano de publicação}

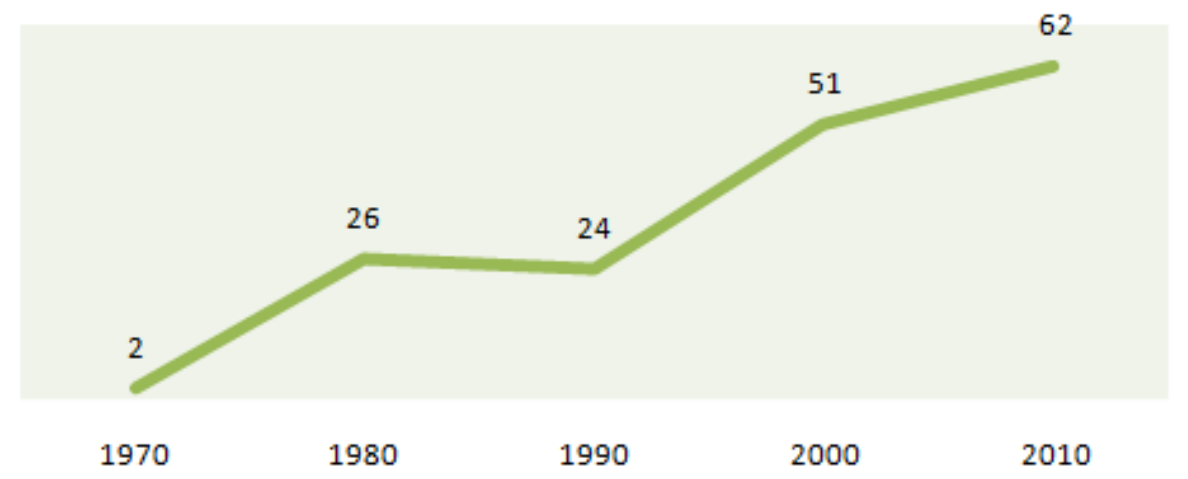

Fonte: Elaborado pelo autor, com base na pesquisa realizada.

A distribuição entre os periódicos se deu de forma bastante diversa, resultando em 52 diferentes periódicos. Destes, os que apresentaram maior número de publicações sobre o assunto foram a) Knitting International: 29 artigos; b) International Journal of Clothing Science and Technology: 15 artigos; c) Textile Month: 14 artigos; d) Journal of the Textile Institute: 13 artigos; e) Journal of Industrial Textiles: 12 artigos; f) Nanoscale: 6 (seis) artigos; g) Measurement Science and Technology; Textiles and Clothing Sustainability; International Journal of Fashion Design, Technology and Education: 5 (cinco) artigos cada; h) International Journal of Innovation and Applied Studies; Materials World; Textile Horizons: 4 (quarto) artigos cada; i) Fibre Chemistry; Journal of Nanomaterials: 3 (três) artigos cada; j) Advances in Textiles Technology; Industry and innovation; International Dyer, Textile Printer, Bleacher and Finisher; Journal of Materials Science: Materials in Medicine; Professional Engineering: 2 (dois) artigos cada; k) Antennas and Wireless Propagation Letters; Apparel International; Architectural Review; Assembly Automation; Biomaterials; Coloration Technology; Composites Manufacturing (VA); Engineering Insight; Evolution; Financial Times; $i$ Manager's Journal on Future Engineering and Technology; Industria Textila; Industrial Management \& Data Systems; International Dyer; International Journal of Designed Objects; International Journal of Environment and Pollution; International Journal of Nanotechnology; International Journal of Technology Management; International Water Power \& Dam Construction; Journal of Biomedical Materials Research, Part B: Applied Biomaterials; Journal of Chemical Technology \& Biotechnology; Journal of Nanoparticle Research; Journal of the Society of Dyers and Colourists; Nature Nanotechnology; Polymer International; Powder Metallurgy; Process Biochemistry; Sports Engineering; Technovation; Textile Research Journal; Times; World Journal of Engineering; World Pumps: 1 (um) artigo cada.

O gráfico 2 apresenta esta distribuição. Os periódicos que apresentaram somente uma publicação foram agrupados sob o título "Outros periódicos". 


\section{Distribuição por periódicos}

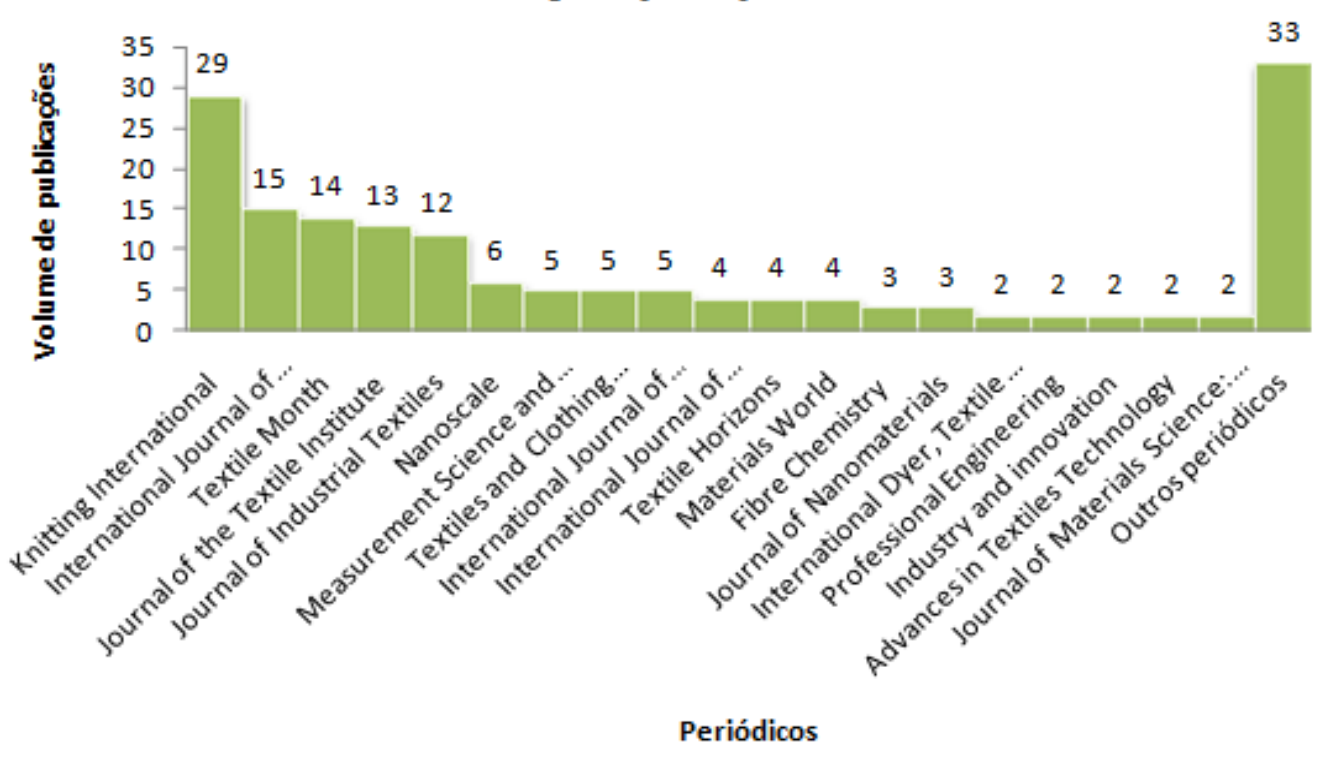

Fonte: Elaborado pelo autor, com base na pesquisa realizada.

\section{CONCLUSÃO}

A revisão bibliométrica realizada foi bem sucedida quando aplicada à base de dados ProQuest. Os resultados foram adequados às premissas determinadas no protocolo. Porém, os mesmos comandos de busca quando aplicados à base de dados Scopus, apresentaram resultados muito elevados, o que levou à uma revisão de alguns dos títulos, a fim de verificar a adequação dos resultados. A partir dessa revisão superficial por títulos percebeu-se que os resultados nessa base apresentavam-se camuflados, pois muitos dos trabalhos não tinham nenhuma relação com os pressupostos desta pesquisa. Assim, sugere-se uma revisão dos termos aplicados à base Scopus, buscando torná-los mais específicos, visto que os resultados totais ultrapassam sete mil trabalhos, o que torna inviável a revisão por títulos.

Entende-se que os processos de inovação têm papel determinante no sucesso das organizações. No setor de Moda, esta afirmação tem ainda mais força, em razão do sistema de atualização dos produtos, adotado atualmente pelo setor. Este desejo constante de atualização resulta na necessidade de estudos que aumentem as chances de aceitação dos produtos pelos consumidores e conseqüentemente diminuam os riscos financeiros. Assim, entram em cena os estudos sobre inovação e também sobre de tendências.

Porém, a partir dos resultados analisados da base ProQuest, é possível perceber que há uma baixa associação entre os termos "Inovação" e "Design de Moda", e boa parte dos artigos encontrados foram publicados em periódicos relacionados à tecnologia têxtil. Portanto, parece haver uma lacuna no desenvolvimento de trabalhos que abordem os temas com foco no setor de confecção. Essa afirmação poderá ser confirmada através de possíveis aprofundamentos do presente estudo, por meio da análise dos artigos. Cabe, dessa forma, dar seguimento à pesquisa selecionando e analisando os trabalhos de fato pertinentes ao tema. 


\section{REFERÊNCIAS}

ABIT - Associação Brasileira da Indústria Têxtil e de Confecção. Monitor ABIT:

Conjuntura da Indústria Têxtil e de Confecção Brasileira. Junho de 2013. Disponível em: http://www.abit.org.br/adm/Arquivo/Servico/020448.pdf. Acesso em: 28 jul. 2013.

BAREGHEH, Anahita; ROWLEY, Jennifer; SAMBROOK, Sally. Towards a multidisciplinary definition of innovation. Management Decision, Vol. 47 Iss: 8, pp. $1323-1339$. Disponível em:http://www.emeraldinsight.com/doi/abs/10.1108/00251740910984578 Acesso em: 01 jun. 2016.

BUFREM, Leilah; PRATES, Yara. O saber científico registrado e as práticas de mensuração da informação. Ciência da Informação, Brasília, v. 34, n. 2, p. 9-25, maio/ago. 2005. Disponível em: http://www.scielo.br/pdf/ci/v34n2/28551 Acesso em: 10 jun. 2016.

CACHON, Gérard P.; SWINNEY, Robert. The Value of Fast Fashion: Quick Response, Enhanced Design, and Strategic Consumer Behavior. Management Science, Vol. 57, No. 4, April 2011, pp. 778-795. Disponível em: http://opim.wharton.upenn.edu/ cachon/pdf/cachon-swinney-2011.pdf. Acesso em: 28 jul. 2013.

CALDAS, Dario. Observatório de sinais: teoria e prática da pesquisa de tendências. Rio de Janeiro: Senac Rio, 2004.

DESIGN BRASIL. Disponível em: http://www.designbrasil.org.br. Acesso em: 01 ago. 2013.

Financiadora de Estudos e Projetos - FINEP (Brasil) (Org.). Manual de Oslo: DIRETRIZES PARA COLETA E INTERPRETAÇÃO DE DADOS SOBRE INOVAÇÃO. 3. ed. OECD, 1997. 184 p. Tradução: Flávia Gouveia - Coordenação editorial: Palmira Moriconi. Disponível em: http://www.finep.gov.br/images/apoio-e-financiamento/manualoslo.pdf. Acesso em: 01 jun. 2016.

GIL, A. Como elaborar projetos de pesquisa. São Paulo: Atlas, 2002.

KELLEY, Thomas; LITTMAN, Jonathan. As 10 faces da inovação: estratégias para turbinar a criatividade. Rio de Janeiro: Elsevier, 2007.

KOTLER, Philip. A bíblia da inovação. São Paulo: Leya, 2011.

LYNN, Gary; REILLY, Richard. Produtos arrasadores: 5 segredos para desenvolver produtos vencedores. Rio de Janeiro: Campus, 2003.

SOUSA, Richard Perassi Luiz de; GOMEZ, Luiz Salomão Ribas; CAMPOS, Amanda Queiroz. O sistema cultural da moda. Alceu, Rio de Janeiro, v. 14, n. 27, p.33-47, 04 nov. 2013. Semestral. Disponível em: <http://revistaalceu.com.pucrio.br/cgi/cgilua.exe/sys/start.htm?infoid=476\&sid=39>. Acesso em: 09 jun. 2016. 